\title{
Linguistic-Cognitive Outcomes in Children with Acute Lymphoid Leukemia: An Exploratory Study
}

\author{
Michelle Miranda Pereira (1D \\ Debora Maria Befi-Lopes \\ Aparecido José Couto Soares (D) \\ Fernanda Chiarion Sassi \\ Claudia Regina Furquim \\ de Andrade
}

School of Medicine, University of São Paulo, São Paulo, Brazil
Correspondence: Aparecido José Couto Soares

School of Medicine, University of São

Paulo, Cipotânea Street, 5I - Cidade

Universitária - São Paulo, Brazil

Tel +55 II 309। -7455

Email ajcsoares@usp.br
Background: Acute lymphoid leukemia (ALL) is the most prevalent cancer of childhood. Impairment in linguistic and memory skills is a possible late sequela in cancer survivors that can limit their quality of life and the overall performance of the individual in society. There is evidence that survivors of ALL treated exclusively with chemotherapy demonstrate significant differences in long-term linguistic and memory functions and also changes in neuroanatomical integrity. However, most studies described do not count on a speech-language pathologist in their team, which we consider important to discuss. Thus, the objective of the present study was to assess memory and vocabulary skills in the pediatric population diagnosed with acute lymphoid leukemia during chemotherapy treatment.

Materials and Methods: An observational cohort study was conducted over a 1.8-year period. Participants of this research were children diagnosed with ALL. All participants were assessed on their linguistic-cognitive skills (ie, vocabulary, short-term memory and lexical access). All data underwent statistical analyses.

Results: The results of the current study found no major significant difference in the linguistic-cognitive performance of children with ALL and their healthy controls. Regarding the linguistic variables, we believe that there should be a differentiation between the effects of the drugs and the effects of social communication skills on performance.

Conclusion: This first characterization of the linguistic-cognitive abilities of children with ALL did not identify differences between these children and their healthy peers, although we were able to identify variables regarding the multidisciplinary team and social communication that should be considered in future studies.

Keywords: interdisciplinary research, leukemia, children, language

\section{Introduction}

Acute lymphoid leukemia (ALL) is the most prevalent cancer of childhood, accounts for $26.8 \%$ of cancer diagnoses among children worldwide and is the most common form in the pediatric population. ${ }^{1,2}$ Impairment in linguistic and memory skills is a possible late sequela in cancer survivors that can limit the quality of life and the overall performance of the individual in society. ${ }^{2,3}$ The historical use of cranial radiation therapy (CRT), followed by intensive chemotherapy treatment of the central nervous system (CNS), has resulted in a 5-year event-free survival rate of approximately $80 \%$ in standard-risk ALL. ${ }^{1}$ However, it is widely reported that these ALL survivors often suffer from long-term neurocognitive deficits that have a negative impact on their health-related quality of life (HRQoL) and daily functioning., ${ }^{3,4}$

There has been a paradigm shift in the treatment strategy for ALL over the past two decades. ${ }^{4,5}$ Although initial success was obtained with prophylactic CRT, this 
approach was gradually replaced by contemporary ALL therapeutic protocols, which consist of intensified intravenous and intrathecal administration of chemotherapeutic drugs for standard risk patients. ${ }^{4-6}$ A recent clinical trial reported that with the elimination of CRT, chemotherapyonly treatment protocols for ALL have resulted in an unprecedented overall survival rate of $93.5 \%{ }^{6}$ Despite these promising results, patients who receive contemporary treatments still experience a myriad of treatmentrelated adverse effects, such as osteonecrosis and cardiovascular and endocrine morbidity. ${ }^{7-9}$

The long-term effects of chemotherapy on linguistic and cognitive functions in children with ALL have received increased attention, especially in the last decade. Direct CNS chemotherapy has replaced cranial irradiation in the treatment of leukemia since the detrimental effects on intellectual function and learning have become evident. However, findings in the literature on the effects of direct chemotherapy on the CNS have not been consistent about the possibility of cognitive deficits and which functions are most affected. ${ }^{10-14}$

Before the 1960s, the prognosis of children with cerebral tumors and ALL was uncertain; thus, concerns regarding late side effects were not worth examining. However, over the last 30 years the survival rate of children with tumors in the CNS has risen sharply due to the increased sophistication of procedures and treatment, and concerns about cognitive and linguistic skills have become increasingly discussed in studies due to the treatment-related neurotoxicity. ${ }^{14}$

In regard to the cognitive impact related to pediatric cancer treatment, it is important to highlight that protocols for treatment recommend medicine therapy that both alone and combined involve the use of potential neurotoxic agents. Thus, cognitive disorders due to such neurotoxic agents and radiotherapy will be dependent on complex interactions of multiple factors, such as the nature, localization and length of the tumor, sociocultural routines of the patient, quality and number of stimuli in their environment, age of diagnosis and CNS maturational processes. ${ }^{14-16}$

The results from published studies suggested that despite the elimination of CRT, survivors who were treated with contemporary protocols still suffered from minor but detectable cognitive impairment. While a handful of studies did not observe a statistically significant difference in levels of cognitive functioning between ALL survivors who received contemporary protocols and noncancer controls or age-matched populations, ${ }^{10-14}$ some studies have found that survivors presented poorer neurocognitive performances than controls. ${ }^{15-20}$ One published study reported that compared with the expected rate of $2 \%$ (predicted rate of healthy controls with scores two standard deviations below the age-based population mean), greater rates of severe impairment were noted in executive function $(15.9 \%)$, attention (14.5\%) and memory (13.1\%) in survivors who received only chemotherapy treatment. ${ }^{14,15}$ Mild but apparent reduced levels of sustained attention and executive functions, such as cognitive flexibility, verbal fluency and attentional flexibility, were reported, and delayed memory and motor functioning were observed in chemotherapy-treated survivors. ${ }^{15-21}$ Hence, there is mounting evidence to suggest that even in the absence of CRT, contemporary protocols might induce an accelerated rate of cognitive decline in survivors.

As widely reported in memory literature, both verbal and short-term memory are fundamental to language development and maintenance not only to expand oral vocabulary but also to support metalinguistic skills and reading and writing acquisition. Moreover, verbal fluency is related to both memory and language itself since it involves not only the recovery of stimuli but also depends on the acquired lexicon. Such skills work together and provide the proper integration of vocabulary, lexicon access and communication skills as a functioning whole. $^{20-23}$

Among studies that involved a cohort of only chemotherapy-treated ALL survivors, there is adequate evidence to show that survivors who received high-dose intravenous methotrexate (MTX) (defined in this review as a single dose of more than $1 \mathrm{~g} / \mathrm{m} 2$ of methotrexate) had more problems in language and memory skills than those given low-dose methotrexate, indicating that neurotoxicity related to methotrexate might be dose-related. ${ }^{20-23}$ It is worth mentioning that one study observed a direct impact of methotrexate on neurocognitive function, such that cumulative doses of intravenous methotrexate increased the risk for slowed processing speed by $3 \%$ for each $1 \mathrm{~g} /$ $\mathrm{m} 2 .{ }^{22,24}$ However, there are limited studies in the literature that compared the neurotoxic intensity of triple intrathecal therapy (ie, cytarabine, methotrexate, hydrocortisone) and intrathecal methotrexate monotherapy; one of the included studies reported no significant differences in cognitive outcomes between these two groups. ${ }^{24,25}$

Studies that indicate neurocognitive deficits associated with chemotherapy in children with leukemia suggest that 
the most affected functions are attention, information processing speed, memory, verbal comprehension, visuospatial and visuomotor skills, as global intellectual function remains relatively preserved. Deficits in linguistic and memory skills are presented as a major issue in leukemia survivors treated with chemotherapy, which may lead to behavioral problems and school struggle. ${ }^{26}$ Age at the time of diagnosis and female gender are indicated as risk factors for more severe cognitive-linguistic sequelae. ${ }^{14,15}$ The use of higher doses of medications is also considered a risk factor, suggesting greater neurotoxicity. ${ }^{15}$

There is evidence that survivors of ALL treated exclusively with chemotherapy demonstrate significant differences in long-term linguistic and memory functions ${ }^{16}$ and changes in neuroanatomical integrity. ${ }^{17]}$ Developmental delays are noted as a consequence of the hospitalization process. One of the affected areas of development is language ${ }^{18-22}$ and functions such as operational memory, processing speed and cognitive efficiency/fluency. ${ }^{23}$

Evidence related to the effects of childhood cancer and its treatment in the CNS (chemotherapy and/or radiotherapy) points to cognitive-linguistic impairments, especially in younger children, emphasizing the importance of assessing these functions throughout the treatment. $3,5,8,24,25$ Based on the literature and considering all the contradictory results around the topic, it is possible to affirm that investigating the linguistic and cognitive development of children with cancer will provide a better characterization of such skills in this population, leading to deeper knowledge in the field. In addition, it is fundamental to consider that most studies described here do not count on a speechlanguage pathologist (SLPs) in their team, which we consider important to discuss. Although SLPs and clinical neuropsychologists engage in some common professional practice, SLPs will analyze the impact of cognitivelinguistic behaviors on communication (ie, oral and written languages). SLPs will provide detailed information on individuals who are having more difficulty with languagebased tasks than with non-language tasks. Members of a multidisciplinary team will benefit from this information because it contributes to the total picture of a child's language, cognitive, sensory-motor and behavioral profile. $^{27}$ Additionally, these data can promote the implementation of more appropriate interventions, attract clinical attention to this population, provide conditions that allow cognitive-linguistic development and school reintegration after treatment, thereby improving quality of life.
Thus, the objective of the present study was to assess memory and vocabulary skills in the pediatric population diagnosed with acute lymphoid leukemia during chemotherapy treatment. Our hypothesis is that children with ALL will present poorer linguistic-cognitive performance when compared to their healthy peers.

\section{Materials and Methods}

The study design was approved by the Ethics Committee for the Analysis of Research Projects of the Institution (CAPPesq HCFMUSP Protocol no. 1.391.342). Prior to their enrollment, all participants and their families were informed of the purpose and procedures of the study, after which all gave written informed consent.

\section{Participants}

An observational cohort study was conducted over a 1.8-year period (April 2016 to December 2017). Although there is a general understanding that children with acute lymphoid leukemia (ALL) who have undergone chemotherapy treatment are likely to present cognitivelinguistic impairments, there is still limited evidence to support this finding. For this reason, we conducted an exploratory study. Participants of this research were children diagnosed with acute lymphoid leukemia (ALL) at the Institute of Cancer for Children of Hospital das Clínicas, School of Medicine of the University of São Paulo, Brazil and who were referred to the Division of Speech-Language Pathology and Oral Myology of the same institution, at the physician's discretion, for a speech/language assessment and possible rehabilitation.

Patients were recruited for participation if they presented the following characteristics: medical diagnosis of ALL; age between 6 and 10 years and 11 months (ie, to isolate possible language development issues); having already undergone the induction phase of chemotherapy treatment, considering that high doses of methotrexate, a drug with high neurotoxicity, ${ }^{15,28-30}$ are given during this phase of treatment. Assessing children after these phases ensured that all of the participants had been exposed to the same risk of drug neurotoxicity. Participants were excluded from the cohort if they were bilingual (ie, only children who spoke Brazilian Portuguese were included in the study), presented any genetic syndromes and/or neurologic disorders and/or auditory impairments, had undergone radiotherapy and/or bone marrow, transplantation, and if they presented any communication deficits or learning complaints that might 
interfere in the understanding of the instructions given during the assessment session.

At our Institution, prior to chemotherapy, children with ALL are classified as having a high or low risk for relapse. The chemotherapy treatment is typically divided in three phases and each phase will last according to the chances of relapse: induction (lasts 4 weeks); consolidation/intensification (lasts between 2 and 8 weeks); and maintenance/ post-consolidation (lasts for about 2 years). In order to better characterize our study sample, we also included a few clinical indicators: age at diagnosis; time between the start of treatment and speech-language assessment (in months) and total duration of hospitalizations (in days); and current phase of the chemotherapy treatment protocol.

For comparison purposes, we included a control group (CG) of healthy volunteers, thoroughly matched by age, gender, years of schooling and intellectual skills (ie, nonverbal intelligence coefficient) to the research group. The controls were selected from among the group of children who attend regular follow-up at the Division of General Pediatrics of the same institution.

\section{Procedures}

All participants were assessed on two consecutive days by the same group of speech-language pathologists. The first session lasted between 20 and 30 minutes and the second session between 45 and 60 minutes. On the first day, to ensure that children in the research group and the control group were properly paired, all participants underwent the following evaluations prior to data gathering:

(a) Auditory functioning - to verify auditory function, field audiometry using a pediatric audiometer was performed. Thresholds were considered normal if the participants presented a response $\leq 30 \mathrm{dBNA}$ at the $500 \mathrm{~Hz}$ frequency and $\leq 20 \mathrm{dBNA}$ at the 1000 , 2000 and $4000 \mathrm{~Hz}$ frequencies.

(b) Intellectual skills - the Raven's Colored Progressive Matrix test ${ }^{31}$ is internationally recognized as a culture reduced test of nonverbal intelligence for younger children. This test has no time limit, and comprises three sets of twelve matrix designs. In this version, each item is printed with a brightly colored background, making the test more appealing to children. The child is shown a series of patterns with parts missing. The missing parts have a simple shape and are below the matrix, among other similarly shaped pieces. The items are arranged in increasing level of difficulty. The child can either point to the missing pattern piece or record its corresponding number on a record form.

The accuracy of the answers was registered and compared to the parameters proposed by the test. The intelligence coefficient was classified as follows: V - much lower than average; IV - below average; III - average; II - above average; I - superior. According to the results on the Raven's Colored Progressive Matrix test, children on the research group were classified as follows: I - superior $\mathrm{n}=3$; II - above average $\mathrm{n}=4$; III - average $\mathrm{n}=10$; IV below average $n=1$. Children on the control group presented the following classification: I - superior $n=7$; II above average $n=5$; III - average $n=6$. The result of the statistical analyses indicated no significant difference between the groups (Pearson's Chi-square test; $\mathrm{p}=0.085$ )

\section{Linguistic-Cognitive Measures}

Data gathering began on the second day, after the children in the research group had been properly matched to their healthy peers. The set of assessments was applied consecutively in all children and followed the same order, as described below:

\section{Expressive Vocabulary}

For the assessment of the expressive vocabulary, we used the vocabulary test from the WISC IV ${ }^{32}$ test. This test comprises 36 items divided in two parts. The first four items are composed by pictures that must be named by the child. One point is given for each picture that is named correctly. The other items are verbal orders involving the description of specific nouns (what is an umbrella?) and verbs (what does abandon mean?). According to the test, the child will receive 0 (zero) points if the answer is inadequate or absent; 1 (one) point if the answer is partially correct and 2 (points) if the answer is completely correct. At the end of the test, the points are added and the performance of the child is classified as: above average, average and below average.

\section{Lexical Access}

In order to evaluate lexical access, we used a semantic verbal fluency test. This test aims to assess the systematic search of elements in a given semantic category. The test was applied following the methodology proposed by Malloy-Diniz et al. ${ }^{33}$ Categories that are relevant to the age range of the participants included in the study were used (ie, animals, body parts and foods). The total number 
of correct words was registered for each category based on the criteria proposed by Charchat-Fichman. ${ }^{34}$

The results were grouped as follows: children who presented the number of correct words $\leq 50 \%$ were classified as I; children who presented the number of correct words between $50 \%$ and $75 \%$ were classified as II; children who presented the number of correct words between $75 \%$ and $90 \%$ were classified as III; and children who presented the number of correct words $>90 \%$ were classified as IV.

\section{Short-Term Memory}

For the assessment of short-term memory, the pseudoword repetition test of Santos ${ }^{35}$ and the WISC IV ${ }^{32}$ digits subtest were used. The pseudoword repetition test ${ }^{35}$ has already been standardized for Brazilian Portuguese speaking children. It is composed of 40 pseudowords, divided according to their similarity to real words of the Brazilian Portuguese language (ie, 10 with low similarity, 20 with average similarity and 10 with high similarity) and to their extension (ie, dissyllable, trisyllable, polysyllable). According to the test, for the target pseudoword to be considered correct, the child must produce it in the exact same manner. Any phonological substitutions are considered errors (ie, phonological disorders are no longer expected in the studied age range). The child's performance is then compared to his or hers schooling level, and classified as a) greater than expected, b) as expected, or c) below expected.

The WISC IV ${ }^{32}$ digits subtest consists of eight series of direct order digits, with a gradual increase in the number of digits in each series. Each item consists of two sets of digits, constituting two attempts. The maximum gross result in the direct order is 16 points. The child's performance is rated as a) greater than expected, b) as expected, or c) below expected. The reverse order digits was administered even if the child completely failed the direct order test. The reverse digit repetition consists of seven series of reverse order digits. Each item is composed of two sets of digits, constituting two attempts. The maximum gross result in the reverse order is of 14 points. The child's performance is rated as a) greater than expected, b) as expected, or c) below expected.

\section{Data Reliability}

In order to evaluate data reliability, all participants were assessed by two experienced speech-language pathologists. The speech-language pathologists assigning the linguistic-cognitive assessments had successfully passed specific training tests. The Kappa Coefficient was used to verify agreement between examiners for the overall scores. The obtained results were as follows: expressive vocabulary $(\mathrm{p}=0.963)$; pseudoword repetition test $(\mathrm{p}=0.955)$; direct order digits test $(\mathrm{p}=0.941)$; inverse order digits test $(p=0.912)$, all indicating a high level of agreement.

\section{Statistical Analysis}

The collected data were submitted to statistical analysis in SPSS software version 25. Due to the number of participants in the sample, nonparametric tests were used for all inferential analyses. The level of significance was 5\%.

For the quantitative variables, descriptive analyses (mean, standard deviation, median, minimum and maximum) and nonparametric inferences were performed using the Mann-Whitney test (for comparisons between groups). For the qualitative variables, descriptive analyses (total and percentage counts) and nonparametric inferences were performed using Pearson's chi-square test (for comparisons between groups).

\section{Results}

After the inclusion criteria were applied, the final study sample consisted of 36 participant - ie, 18 patients with ALL and 18 healthy controls. Regarding the phases of chemotherapy treatment, 5 children were in the intensification phase; 3 children were in the late consolidation phase and 10 children were in the maintenance phase. Table 1 presents the clinical characteristics for the participants with ALL.

Table I Characterization of Children with Acute Lymphoid Leukemia $(n=18)$

\begin{tabular}{|c|c|c|c|c|c|}
\hline & Mean & SD & Minimum & Median & Maximum \\
\hline Age (in years) & 8.9 & 1.5 & 6.6 & 8.9 & 11.3 \\
\hline Age at diagnoses & 7.7 & 1.9 & 4.4 & 7.8 & 10.3 \\
\hline Duration of treatment (in months) & 15.7 & 9.6 & 5.0 & 12.5 & 34.0 \\
\hline Number of hospitalizations during treatment & 3.3 & 2.3 & 0.0 & 3.0 & 8.0 \\
\hline Total duration of hospitalizations (in days) & 30.2 & 21.3 & 0.0 & 29.5 & 85.0 \\
\hline
\end{tabular}


Table 2 shows the results of the variables used to pair children in ALL to the control group.

The results obtained on the vocabulary test were compared between the groups. The statistical analyses indicated no significant difference for any of the variables (Table 3).

The results of the verbal fluency assessment were also compared between the groups. An isolated difference between the groups was found for "body parts" (ie, children in the control group presented a better performance). (Table 4).

The results on the digit repetition tests (ie, direct and reverse order) are shown in Table 5. An isolated difference between the groups was observed in the reverse order digit test, having an influence on the and for the gross score (ie, children in the control group presented a better performance).

Results in Table 6 show the between-group comparison for the results on the pseudoword repetition test, no significant differences were observed when comparing the performance of both groups.

\section{Discussion}

The results of the current study found no major significant difference in the linguistic-cognitive performance of children with ALL and their healthy controls. Our findings are consistent with some of the results already reported in the literature. ${ }^{14,36,37}$ Our results suggest that chemotherapy treatment does not seem to affect, in a short period of time, language development and other cognitive abilities.

The existence of memory deficits in children with leukemia has already been explored in different studies. ${ }^{38-42}$ It is important to highlight, however, that most of these studies were conducted with children/adolescents after ceasing their treatment, thus indicating the late effects of chemotherapy on neurocognitive skills, including memory. Moreover, some of these studies assessed memory using different protocols, making it difficult to generalize findings. Recently, a meta-

Table 2 Variables Used to Match Children with Acute Lymphoid Leukemia and Healthy Peers

\begin{tabular}{|c|c|c|c|}
\hline & ALL $(n=18)$ & CG $(n=18)$ & $p$-value \\
\hline \multirow{6}{*}{$\begin{array}{l}\text { Schooling } \\
\text { Total number (\%) }\end{array}$} & Ist year $=4(22.2 \%)$ & Ist year $=2(1|| \%)$. & \multirow[t]{6}{*}{0.801} \\
\hline & 2 nd year $=2(11.1 \%)$ & 2 nd year $=5(27.8 \%)$ & \\
\hline & $3 r d$ year $=5(27.8 \%)$ & $3 r d$ year $=3(16.7 \%)$ & \\
\hline & 4 th year $=2(11.1 \%)$ & 4 th year $=4(22.2 \%)$ & \\
\hline & 5 th year $=3(16.7 \%)$ & 5 th year $=3(16.7 \%)$ & \\
\hline & 6 th year $=2(11.1 \%)$ & 6 th year $=1(5.6 \%)$ & \\
\hline \multirow{4}{*}{$\begin{array}{l}\text { Performance in the Raven IQ test } \\
\text { Total number (\%) }\end{array}$} & Superior $=3(16.7 \%)$ & Superior $=7$ (38.9\%) & \multirow[t]{4}{*}{0.085} \\
\hline & Above average $=4(22.2 \%)$ & Above average $=5(27.8 \%)$ & \\
\hline & Average $=10(55.6 \%)$ & Average $=6(33.3 \%)$ & \\
\hline & Below average $=I(5.6 \%)$ & Below average $=0(0 \%)$ & \\
\hline
\end{tabular}

Note: Pearson's chi-square test.

Abbreviations: ALL, acute lymphoid leukemia; CG, control group.

Table 3 Between-Group Comparison for the Results on the Expressive Vocabulary Test

\begin{tabular}{|l|c|c|c|}
\hline Vocabulary Test WISC - IV & ALL $(\mathbf{n}=\mathbf{~ 1 8})$ & CG $(\mathbf{n}=\mathbf{~ 1 8})$ & p-value \\
\hline $\begin{array}{l}\text { Score } \\
\text { Mean }( \pm S D)\end{array}$ & $25.6( \pm 9.3)$ & $25.5( \pm 5.5)$ & 0.913 \\
\hline $\begin{array}{l}\text { Performance classification } \\
\text { Total number }(\%)\end{array}$ & Greater than expected $=6(33.3 \%)$ & Greater than expected $=7(38.9 \%)$ & 0.988 \\
\cline { 2 - 4 } & Within expected $=7(38.9 \%)$ & Within expected $=5(27.8 \%)$ \\
\cline { 2 - 4 } & Below expected $=5(27.8 \%)$ & Below expected $=6(33.3 \%)$ \\
\hline
\end{tabular}

Notes: Mann-Whitney test (score); Pearson's chi-square test (performance classification).

Abbreviations: ALL, acute lymphoid leukemia; CG, control group. 
Table 4 Group Comparison for the Results on the Lexical Access Test

\begin{tabular}{|c|c|c|c|c|}
\hline \multicolumn{2}{|l|}{ Semantic Verbal Fluency } & \multirow{2}{*}{$\begin{array}{c}\text { ALL }(\mathbf{n}=18) \\
12.1( \pm 4.9)\end{array}$} & \multirow{2}{*}{$\frac{C G(n=18)}{14.7( \pm 4.3)}$} & \multirow{2}{*}{$\frac{p \text {-value }}{0.111}$} \\
\hline Score & Animals & & & \\
\hline Mean ( \pm standard deviation) & Body parts & $13.0( \pm 3.8)$ & $15.7( \pm 2.7)$ & $0.029 *$ \\
\hline & Food & $12.0( \pm 4.4)$ & $14.0( \pm 3.9)$ & 0.339 \\
\hline \multirow{12}{*}{$\begin{array}{l}\text { Performance } \\
\text { classification } \\
\text { Total number (\%) }\end{array}$} & \multirow[t]{4}{*}{ Animals } & $<50 \%=12(66.7 \%)$ & $<50 \%=8(44.4 \%)$ & \multirow[t]{4}{*}{0.265} \\
\hline & & $50-75 \%=2(11.1 \%)$ & $50-75 \%=4(22.2 \%)$ & \\
\hline & & $75-90 \%=2(11.1 \%)$ & $75-90 \%=2(11.1 \%)$ & \\
\hline & & $>90 \%=2(11.1 \%)$ & $>90 \%=4(22.2 \%)$ & \\
\hline & \multirow[t]{4}{*}{ Body parts } & $<50 \%=13(72.2 \%)$ & $<50 \%=7(38.9 \%)$ & \multirow[t]{4}{*}{0.079} \\
\hline & & $50-75 \%=3(16.7 \%)$ & $50-75 \%=5(27.8 \%)$ & \\
\hline & & $75-90 \%=1(5.6 \%)$ & $75-90 \%=5(27.8 \%)$ & \\
\hline & & $>90 \%=\mathrm{I}(5.6 \%)$ & $>90 \%=\mathrm{I}(5.6 \%)$ & \\
\hline & \multirow[t]{4}{*}{ Food } & $<50 \%=11(61.1 \%)$ & $<50 \%=8(44.4 \%)$ & \multirow[t]{4}{*}{0.308} \\
\hline & & $50-75 \%=6(33.3 \%)$ & $50-75 \%=7(38.9 \%)$ & \\
\hline & & $75-90 \%=I(5.6 \%)$ & $75-90 \%=3(16.7 \%)$ & \\
\hline & & $>90 \%=0(0 \%)$ & $>90 \%=0(0 \%)$ & \\
\hline
\end{tabular}

Notes: Mann-Whitney test (score); Pearson's chi-square test (performance classification); *significant difference according to the Mann-Whitney test. Abbreviations: ALL, acute lymphoid leukemia; CG, control group.

analysis presented an overview of studies that investigated ALL survivors. ${ }^{41}$ In this analysis, it was possible to identify a few studies that adopted the same assessment procedures described in the present investigation. Overall, these specific studies reported poorer working memory in ALL survivors when compared to a control group of healthy individuals.

Another important consideration is the fact that none of studies we found in the literature analyzed the possible correlations between neurocognitive, language and social skills. ${ }^{38-43}$ According to the literature, such skills are highly correlated to oral language proficiency and sophistication (eg, discursive skills) and to underlying skills of reading and writing development, such as phonological awareness. ${ }^{33-35}$ Considering the reported late effects of chemotherapy treatment and how its dynamics can lead to absences from school, a long-term follow-up of the impact on those variables seems to be crucial. Therefore, developing early training intervention programs for memory, language and communications skills throughout the entire treatment protocol is important to reduce such side effects in this population.
Considering the above arguments, our study was, therefore, exploratory and characterized children with ALL who were being treated in a hospital setting. The tests that were chosen for the investigation of these children were very basic and easy to apply in two assessment sessions, avoiding that the children would stop responding due to loss of attention. However, this set of tests were not able to point differences in the linguistic-cognitive performance of children with ALL and their healthy controls.

During the course of the research, we were able to identify variables that had not been considered at the design of the study. The first of these variables was the difficulty to interact with the multidisciplinary team. On several occasions the oncology team, who was responsible for the administration of the treatment drugs, had to include different drugs or modify the duration of the cycle of the chemotherapy treatment due to clinical response and side effects. These alterations during the data gathering period were not informed to the field researchers. While it is reasonable to understand why changes were made to the ongoing treatment protocol, these alterations must be considered in future studies as 
Table 5 Group Comparison for the Results on the Digit Repetition Tests

\begin{tabular}{|c|c|c|c|c|}
\hline \multicolumn{2}{|l|}{ Digit Repetition Tests } & \multirow{2}{*}{$\frac{\text { ALL }(n=18)}{6.1( \pm 1.7)}$} & \multirow{2}{*}{$\frac{C G(n=18)}{6.6( \pm 1.6)}$} & \multirow{2}{*}{$\frac{p \text {-value }}{0.226}$} \\
\hline Maximum number of & Direct order & & & \\
\hline $\begin{array}{l}\text { digits } \\
\text { Mean ( } \pm S D)\end{array}$ & Reverse order & $4.2( \pm 1.8)$ & $5.7( \pm 1.5)$ & $0.029 *$ \\
\hline & Gross score & $10,3( \pm 2,7)$ & $12,3( \pm 2,8)$ & $0.017^{*}$ \\
\hline \multirow{9}{*}{$\begin{array}{l}\text { Performance } \\
\text { classification } \\
\text { Total number (\%) }\end{array}$} & \multirow[t]{3}{*}{$\begin{array}{l}\text { Repeated digits in direct } \\
\text { order }\end{array}$} & $\begin{array}{c}\text { Greater than expected }=3 \\
(16.7 \%)\end{array}$ & $\begin{array}{c}\text { Greater than expected }=8 \\
(44.4 \%)\end{array}$ & \multirow[t]{3}{*}{0.462} \\
\hline & & Within expected $=3(16.7 \%)$ & Within expected $=0(0 \%)$ & \\
\hline & & Below expected $=12(66.7 \%)$ & Below expected $=10(55.6 \%)$ & \\
\hline & \multirow[t]{3}{*}{$\begin{array}{l}\text { Repeated digits in reverse } \\
\text { order }\end{array}$} & $\begin{array}{c}\text { Greater than expected }=4 \\
(22.2 \%)\end{array}$ & $\begin{array}{c}\text { Greater than expected }=6 \\
(33.3 \%)\end{array}$ & \multirow[t]{3}{*}{0.104} \\
\hline & & Within expected $=0(0 \%)$ & Within expected $=2(11.1 \%)$ & \\
\hline & & Below expected $=14(77,8 \%)$ & Below expected $=10(55.6 \%)$ & \\
\hline & \multirow[t]{3}{*}{ Gross score } & $\begin{array}{c}\text { Greater than expected }=3 \\
(16.7 \%)\end{array}$ & $\begin{array}{c}\text { Greater than expected }=9 \\
(50.0 \%)\end{array}$ & \multirow[t]{3}{*}{0.279} \\
\hline & & Within expected $=2(11.1 \%)$ & Within expected $=I(5.6 \%)$ & \\
\hline & & Below expected $=13(72.2 \%)$ & Below expected $=8(44.4 \%)$ & \\
\hline
\end{tabular}

Notes: Mann-Whitney test (maximum number of digits); Pearson's chi-square test (performance classification); *significant difference according to the Mann-Whitney test. Abbreviations: ALL, acute lymphoid leukemia; CG, control group.

they are significant and can have a relevant impact on the children's performance. Another point that should be taken into consideration is the child's current cycle of chemotherapy, as the timing of the signs and symptoms in terms of the impacts of toxicity and side effects of the drugs that are used can show differences along the course of treatment - ie, early ( 0 to 3 days); immediate (7 to 21 days) and late (after 21 days). This variable should also be considered in future studies.

Regarding the linguistic variables, we believe that there should be a differentiation between the effects of the drugs and the effects of social communication skills on performance. Considering that the tests we adopted were not able to differentiate children with ALL and their healthy controls, we suggest for future studies (ie, with children above 7 years of age, who have already surpassed the phase of language acquisition and development) the inclusion of social communication tests. Social communication is the use of language in social contexts. It encompasses social interaction, social cognition, pragmatics, and language processing. ${ }^{44}$ All of these tests should be applied prior to the beginning of the chemotherapy treatment in order to establish a baseline on social communication skills. This type of testing would be more sensitive as it includes the assessment of the ability to vary speech style, take the perspective of others, understand and appropriately use the rules for verbal and nonverbal communication, and use the structural aspects of language (eg, vocabulary, syntax, and phonology). ${ }^{44,45}$ All of these tests are used for the distinct diagnosis of social communication impairment and for the identification of cooccurring conditions, considering the effects on intellectual disability; developmental disabilities; learning disabilities; spoken language disorders; written language disorders; learning disabilities; traumatic brain injury aphasia; and right-hemisphere damage. Besides that, future studies should also include information on parenting that could have an impact on the verbal skills of children with ALL and a long-term follow-up in order to investigate predictors of communication outcomes of verbal and nonverbal cognitive abilities. Social environment and family education levels have been pointed as crucial factors for language acquisition and vocabulary development. ${ }^{46,47}$

The treatment of children with cancer has an impact and comprises specific needs - ie, clinical, communication, psychological and social -, needs that would not be considered in the everyday life of children and their family when in typical conditions. The multidisciplinary work involved in 
Table 6 Between-Group Comparison for the Results on Pseudo-Word Repetition Test

\begin{tabular}{|c|c|c|c|c|}
\hline & & ALL $(n=18)$ & CG $(n=18)$ & $p$-value \\
\hline \multirow{4}{*}{$\begin{array}{l}\text { Percentage of correct } \\
\text { answers } \\
\text { Mean }( \pm S D)\end{array}$} & Low similarity & $90.0( \pm 9.1)$ & $94.4( \pm 10.4)$ & 0.091 \\
\hline & Medium similarity & $89.4( \pm 7.5)$ & $92.5( \pm 7.7)$ & 0.192 \\
\hline & High similarity & $97.2( \pm 4.6)$ & $98.3( \pm 5.1)$ & 0.443 \\
\hline & Total & $91.4( \pm 5.2)$ & $94.7( \pm 4.4)$ & 0.055 \\
\hline \multicolumn{2}{|l|}{$\begin{array}{l}\text { Number of correct answers } \\
\text { Mean }( \pm S D)\end{array}$} & $36.6( \pm 2.1)$ & $37.9( \pm 1.8)$ & 0.068 \\
\hline \multirow{3}{*}{\multicolumn{2}{|c|}{$\begin{array}{l}\text { Performance classification } \\
\text { Total number (\%) }\end{array}$}} & Greater than expected $=4(22.2 \%)$ & Greater than expected $=10(55.6 \%)$ & \multirow[t]{3}{*}{0.068} \\
\hline & & Within expected $=13$ (72.2\%) & Within expected = 8 (44.4\%) & \\
\hline & & Below expected $=$ I $(5.6 \%)$ & Below expected $=0(0 \%)$ & \\
\hline
\end{tabular}

Notes: Mann-Whitney test (percentage and number of correct answers); Pearson's chi-square test (performance classification).

Abbreviations: ALL, acute lymphoid leukemia; CG, control group.

caring for children with ALL does not only aim at treating the disease itself, but is an effort to give a much broader assistance to the children and their families. The ability to communicate - verbally and nonverbally - enables the active engagement and building independence in natural communication environments. Communication can decrease the negative and aggressive effects of chemotherapy, allowing children to interact with their peers, play, express their joys, anguishes, anxieties and fears.

Finally, we believe that future studies would benefit from a multicenter collaboration, as it would result in higher rates of patient enrolment and in clearer and more generalizable findings. A multicenter approach would allow important analyses such as gender differences and possible associations of verbal performance with gender, diagnosis and treatment variables will be possible.

\section{Conclusions}

This first characterization of the linguistic-cognitive abilities of children with ALL did not identify differences between these children and their healthy peers. We were able to identify variables regarding the multidisciplinary team and social communication that should be considered in future studies. These variables will allow the investigation of which linguistic-cognitive outcomes are resultant of the neurotoxicity of the drugs used in chemotherapy treatment and which outcomes are resultant of a social communication deprivation, experienced by many of these children in their natural environment.

\section{Author Contributions}

All authors made a significant contribution to the work reported, whether that is in the conception, study design, execution, acquisition of data, analysis and interpretation, or in all these areas; took part in drafting, revising or critically reviewing the article; gave final approval of the version to be published; have agreed on the journal to which the article has been submitted; and agree to be accountable for all aspects of the work.

\section{Disclosure}

The authors have no conflicts of interest to declare. This study was conducted in accordance with the Declaration of Helsinki.

\section{References}

1. Kaatsch P. Epidemiology of childhood cancer. Epidemiology of childhood cancer. Cancer Treat Rev. 2010;36(4):277-285. doi:10.1016/j. ctrv.2010.02.003

2. Zebrack BJ, Zeltzer LK, Whitton J, et al. Psychological outcomes in longterm survivors of childhood leukemia, Hodgkin's disease, and nonHodgkin's lymphoma: a report from the Childhood Cancer Survivor Study. Pediatri. 2002;110(1):42-52. doi:10.1542/peds.110.1.42

3. Anderson FS, Kunin-Batson AS. Neurocognitive late effects of chemotherapy in children: the past 10 years of research on brain structure and function. Pediatr Blood Cancer. 2009;25(2):159-164. doi:10.1002/pbc. 21700

4. Kadan-Lottick NS, Zeltzer LK, Liu Q, et al. Neurocognitive functioning in adult survivors of childhood non-central nervous system cancers. J Natl Cancer Inst. 2010;102(12):881-893. doi:10.1093/jnci/djq156

5. Armstrong GT, Liu Q, Yasui Y, et al. Long-term outcomes among adult survivors of childhood central nervous system malignancies in the childhood cancer survivor Study. J Natl Cancer Inst. 2009;101 (13):946-958. doi:10.1093/jnci/djp148 
6. Ellenberg L, Liu Q, Gioia G, et al. Neurocognitive status in long-term survivors of childhood CNS malignancies: a report from the childhood cancer survivor Study. Neuropsychology. 2009;23(6):705-717. doi:10.1037/a0016674

7. Kesler SR, Lacayo NJ, Jo B. A pilot study of an online cognitive rehabilitation program for executive function skills in children with cancer-related brain injury. Brain Inj. 2011;25(1):101-112. doi:10.3109/02699052.2010.536194

8. Ortiz LCM Classe hospitalar: reflexões sobre sua práxis educativa. Dissertação de Mestrado em Educação [dissertation]. Rio Grande do Sul: Universidade Federal de Santa Maria; 2002.

9. Ullrich NJ, Embry L. Neurocognitive dysfunction in survivors of childhood brain tumors. Semin Pediatr Neurol. 2012;19(1):35-42. doi:10.1016/j.spen.2012.02.014

10. Santos MZ, Júnior JJS, Menezes M. Avaliação do desenvolvimento cognitivo de crianças com câncer por meio do DFH III. Aval Psicol. 2013;12(3):325-332.

11. Palmer SL, Reddick WE, Gajjar A. Understanding the cognitive impact on children who are treated for medulloblastoma. J Pediatr Psychol. 2007;32(9):1040-1104. doi:10.1093/jpepsy/js1056

12. Qaddoumi I, Carey SS, Conklin H, et al. Characterization, treatment, and outcome of intracranial neoplasms in the first 120 days of life. J Child Neurol. 2011;26(8):988-994. doi:10.1177/0883073811401398

13. Instituto Nacional de Câncer. ABC Do Câncer: Abordagens Básicas Para o Controle Do Câncer/Instituto Nacional De Câncer José Alencar Gomes Da Silva. 6th ed. rev.atual - Rio de Janeiro: INCA; 2020.

14. Instituto Nacional de Câncer José Alencar Gomes da Silva. Coordenação de Prevenção e Vigilância. In: Estimativa 2016: Incidência De Câncer No Brasil. Rio de Janeiro: INCA; 2015:56-57.

15. Buizer AI, Sonneville LMJ, Veerman AJP. Effects of chemotherapy on neurocognitive function in children with acute lymphoblastic leukemia: a critical Review of the literature. Pediatr Blood Cancer. 2009;52(4):447-454. doi:10.1002/pbc.21869

16. Cheung YT, Krull KR. Neurocognitive outcomes in long-term survivors of childhood acute lymphoblastic leukemia treated on contemporary treatment protocols: a systematic review. Neurosci Biobehav Rev. 2015;53(2):108-120. doi:10.1016/j.neubiorev.2015.03.016

17. Kim SJ, Park MH, Lee JW, et al. Neurocognitive outcome in survivors of childhood acute lymphoblastic leukemia: experience of a tertiary care hospital in Korea. J Korean Med Sci. 2015;30 (4):463-469. doi:10.3346/jkms.2015.30.4.463

18. Edelmann MN, Krul KR, Liu W, et al. Diffusion tensor imaging and neurocognition in survivors of childhood acute lymphoblastic leukaemia. Brain. 2014;137(11):2973-2983. doi:10.1093/brain/ awu230

19. Carvalho MMJ. Introdução A Psiconcologia. 1st ed. Campinas: Livro Pleno; 2013.

20. Lopes LF, Camargo B, Bianchi A. Os efeitos tardios do tratamento do câncer infantil. Rev Ass Med Brasil. 2000;46(3):277-284. doi:10.1590/S0104-42302000000300014

21. Taverna L, Tremolada M, Bonichini S, et al. Motor skill delays in pre-school children with leukemia one year after treatment: hematopoietic stem cell transplantation therapy as an important risk factor. PLoS One. 2017;12(10):1-14. doi:10.1371/journal.pone.0186787

22. Munhoz MA, Ortiz LCM. Um estudo da aprendizagem e desenvolvimento de crianças em situação de internação hospitalar. Rev Educ. 2006;58(1):65-83.

23. Rezende AM, Santos PP, Cerqueira ACM, et al. A criança e o adolescente com câncer em casa de apoio: projetando vivências. Rev SBPH. 2013;16(1):3.

24. Winter AL, Conklin HM, Tyc VL, et al. Executive function late effects in survivors of pediatric brain tumors and acute lymphoblastic leukemia. $J$ Clin Exp Neuropsychol. 2014;36(8):818-830. doi:10.1080/13803395.2014.943695
25. Cristofani LM, Kuczynski E, Odone Filho V. Qualidade de vida nas leucemias na infância. In: FAssumpção Junior FB, Kuczynski E, editors. Qualidade De Vida Na Infância e Na Adolescência. Porto Alegre: Artmed; 2010:137-159.

26. Tremolada M, Taverna L, Bonichini S, et al. Paediatric patients treated for leukemia back to school: a mixed-method analysis about daily life and illness experience. Behav Sci. 2020;10(7):1-15. doi: $10.3390 / \mathrm{bs} 10070107$

27. American Speech-Language-Hearing Association. The role of speech-language pathologists in the habilitation and rehabilitation of cognitively impaired individuals: a report of the subcommittee on language and cognition. Asha. 1987;29:53-55.

28. Vlcková I, Pavelková K, Kepák T, et al. [Changes of neurocognitive functions as result of cancer treatment in children and adolescents]. Klin Onkol. 2008;21(5):294-302. [Indonesian].

29. Iuvone L, Peruzzi L, Colosimo C, et al. Pretreatment neuropsychological deficits in children with brain tumors. Neuro Oncol. 2011;13 (5):517-524. doi:10.1093/neuonc/nor013

30. Chidambaram S, Seshachalam A, Elagovan V, et al. Immediate treatment effects of high-dose methotrexate and cranial irradiation on neuropsychological functions of children treated for acute lymphoblastic leukemia at a regional cancer center. Indian $\mathrm{J}$ Med Paediatr Oncol. 2014;35(4):281-287. doi:10.4103/09715851.144990

31. Raven J, Court J. Coloured Progressive Matrices. London: H. K. Lewis; 1986.

32. Wechsler D. WISC-IV Technical and Interpretive Manual. TX: Psychological Corporation; 2003.

33. Malloy-Diniz LF, Bentes RC, Figueiredo D, et al. Normalización de una batería de tests para evaluar las habilidades de comprensión del lenguaje, fluidez verbal y denominación en niños brasileños de 7 a 10 años: resultados preliminares. Rev Neurol. 2007;44(5):275-280.

34. Charchat-Fichman HC, Oliveira RM, Silva AM. Performance of Brazilian children on phonemic and semantic verbal fluency tasks. Dement Neuropsychol. 2011;5(2):78-84. doi:10.1590/S198057642011DN05020004

35. Santos FH, Bueno OFA, Gathercole SE. Errors in nonword repetition: bridging short- and long-term memory. Braz J Med Biol Res. 2006;39 (3):371-385. doi:10.1590/S0100-879X2006000300008

36. Lewis FM, Perry ML, Murdoch BE. Longitudinal language outcomes following intrathecal chemotherapy for acute lymphoblastic leukaemia. Int $J$ Speech Lang Pathol. 2013;15(2):156-164. doi:10.3109/17549507.2012.684888

37. American Speech-Language-Hearing Association. Scope of practice in speech-language-pathology [Scope of Practice]; 2016. Available from: www.asha.org/policy/. Accessed May 17, 2021.

38. Walsh KS, Paltin I, Goia GA, et al. Everyday executive function in standard-risk acute lymphoblastic leukemia survivors. Child Neuropsychol. 2015;21(1):78-89. doi:10.1080/09297049.2013.876491

39. Cheng YT, Sabin ND, Reddick WE, et al. Leukoencephalopathy and long-term neurobehavioral, neurocognitive and brain imaging outcomes in survivors of childhood acute lymphoblastic leukemia treated with chemotherapy: a longitudinal analysis. Lancet Haematol. 2016;3(10):456-466. doi:10.1016/S2352-3026(16)30110-7

40. Plas EVD, Erdman L, Nieaman BJ. Characterizing neurocognitive late effects in childhood leukemia survivors using a combination of neuropsychological and cognitive neuroscience measures. Child Neuropsychol. 2018;24(8):999-1014. doi:10.1080/09297049. 2017.1386170

41. Iyer NS, Balsamo LM, Bracken MB, et al. Chemotherapy-only treatment effects on long-term neurocognitive functioning in childhood ALL survivors: a review and meta-analysis. Blood. 2015;125(3):353.

42. Espy KA, Moore IM, Kaufmann PM, et al. Chemotherapeutic CNS prophylaxis and neuropsychologic change in children with acute lymphoblastic leukemia: a prospective Study. J Pediatr Psychol. 2001;26(1):1-9. doi:10.1093/jpepsy/26.1.1 
43. Campbell LK, Scaduto M, Slyke DV, et al. Executive function, coping, and behavior in survivors of childhood acute lymphocytic leukemia. J Pediatric Psychol. 2009;34(3):317-327. doi:10.1093/ jpepsy/jsn080

44. Adams C, Lockton E, Freed J, et al. The social communication intervention project: a randomized controlled trial of the effectiveness of speech and language therapy for school-age children who have pragmatic and social communication problems with or without autism spectrum disorder. Int $J$ Lang Commun Disord. 2021;47 (3):233-244. doi:10.1111/j.1460-6984.2011.00146.x

45. American Psychiatric Association. Diagnostic and Statistical Manual of Mental Disorders. 5th ed. Washington, DC: Author; 2013.
46. Nguyen T-N-N, Spencer-Smith M, Pascoe L, et al. Language Skills in Children Born Preterm ( $<30$ Wks'Gestation) Throughout Childhood: Associations With Biological and Socioenvironmental Factors. J Dev Behav Pediatr. 2019;40(9):735. doi:10.1097/DBP.000000 0000000742

47. Setenac M, Johnson S, Charkaluk ML, et al. Maternal education and language development at 2 years corrected age in children born very preterm: results from a European population-based cohort study. J Epidemiol Community Health. 2020;74(4):346-353. doi:10.1136/ jech-2019-213564
Journal of Multidisciplinary Healthcare

\section{Publish your work in this journal}

The Journal of Multidisciplinary Healthcare is an international, peerreviewed open-access journal that aims to represent and publish research in healthcare areas delivered by practitioners of different disciplines. This includes studies and reviews conducted by multidisciplinary teams as well as research which evaluates the results or conduct of such teams or healthcare processes in general. The journal
Dovepress

covers a very wide range of areas and welcomes submissions from practitioners at all levels, from all over the world. The manuscript management system is completely online and includes a very quick and fair peer-review system. Visit http://www.dovepress.com/testimonials. php to read real quotes from published authors. 